\title{
Identification of genes associated with the increased number of four-seed pods in soybean (Glycine max L.) using transcriptome analysis
}

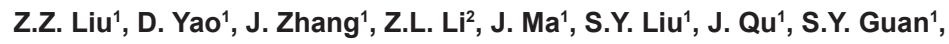 \\ D.D. Wang ${ }^{1}$, L.D. Pan ${ }^{1}$, D. Wang ${ }^{1}$ and P.W. Wang ${ }^{1}$ \\ ${ }^{1}$ Center for Plant Biotechnology, Jilin Agricultural University, Changchun, China \\ ${ }^{2}$ Center for Applied Genetic Technologies, University of Georgia, Athens, GA, USA \\ *These authors contributed equally to this study. \\ Corresponding author: P.W. Wang \\ E-mail: davidmedsci@sina.com
}

Genet. Mol. Res. 14 (4): 18895-18912 (2015)

Received August 16, 2015

Accepted October 9, 2015

Published December 28, 2015

DOI http://dx.doi.org/10.4238/2015.December.28.39

\begin{abstract}
Seed number per pod is an important component of yield traits in soybean (Glycine max L.). In 2010, we identified a natural mutant with an increased number of four-seed pods from a soybean variety named 'Jinong 18' (JN18). Subsequent observations indicated that the trait was stably inherited. To identify and understand the function of genes associated with this mutant trait, we analyzed the genetic differences between the mutant (JN18MT01) and source variety (JN18) by transcriptome sequencing. Three types of tissues, axillary buds, unfertilized ovaries, and young pods at three different growth stages, V6, R1, and R3, were analyzed, respectively. The sequencing results yielded 55,582 expressed genes and 4183 differentially expressed genes (DEGs). Among these, the log2 ratio value of 162 DEGs was $>10$, and 13 DEGs hadoverlapping expression at three different growth stages. Comparisons of DEGs among three different growth stages yielded similar results in terms of the percentage of genes classified into each gene ontology (GO) category. DEGs were classified into 25 different functional groups in clusters of orthologous groups analysis. Proportions of the main functional genes differed significantly over developmental stages.
\end{abstract}


A comparison of enriched pathways among the three developmental stages revealed that 646 unigenes were involved in 103 metabolic pathways. These results show that the development of four-seed pods is associated with a complex network involving multiple physiological and metabolic pathways. This study lays the foundation for further research on cloning and on the molecular regulation of genes related to the four-seed pod mutation.

Key words: Soybean; Four-seed pod mutant; Transcriptome analysis; High-throughput Illumina sequencing; Developmental stages

\section{INTRODUCTION}

Soybean (Glycine max) is a valuable food and forage crop and an important source of high-quality plant protein, oil, and various functional medical and health products (Zhai, 1988). This crop plays an important role in food and economic security, especially in developing countries. Despite the importance of soybean resources, the soybean crop improvement program lags behind those of cereals (Graham and Vance, 2003). One of the main reasons for this lag is a lack of sufficient genomic resources. However, with the recent development of effective and highthroughput sequencing technologies, research on soybean genomics has attracted widespread attention (Cannon et al., 2009; Severin et al., 2010; Chan et al., 2012). Full genome sequencing has been completed for five legumes; soybean, lotus, Medicago, pigeon pea, and chickpea (Sato et al., 2008; Schmutz et al., 2010; Varshney et al., 2011; Young et al., 2011; Jain et al., 2013; Varshney et al., 2013). However, researchers are now faced with the more difficult problem of trying to understand the relationships between genome sequences and molecular function and regulation in different cells. These relationships will directly affect the process of crop improvement.

Under conditions where the external environment and climate do not affect yield, the main factors affecting soybean yield are the number of pods per plant, number of seeds per plant, number of seeds per pod, and 100-seed weight. Previous research has shown that the number of four-seed pods is positively correlated with the number of seeds per pod in soybean, and varieties with a higher proportion of four-seed pods show higher yields and productivity (Peng et al., 1994). A previous study showed that the number of three-seed pods was positively correlated with the yield per plant, and that lines with more seeds per pod produced higher yields than lines with fewer seeds per pod (Zhou et al., 2005). A genetic linkage map was constructed using recombinant inbred soybean lines, and the gene controlling the number of seeds per pod was located on a linkage group. Although there were discrepancies in the heritability of different yield traits, the number of seeds per pod showed high heritability $(80.07 \%)$. That study also showed that the gene controlling the number of seeds per pod was located at the main effective quantitative trait locus that strongly contributed to yield (Wang et al., 2007). Therefore, the number of seeds per pod has some practical value for increasing yield, and increasing the number of seeds per pod may be an effective way to improve yield.

Transcriptome sequencing is an effective strategy used to explore the relationship between gene sequences and molecular mechanisms (Wang et al., 2009; Ozsolak and Milos, 2011; Jain, 2012). Global transcriptome analyses can provide insight into the location of genes, their functions, transcriptional regulation, and the molecular basis of various cellular processes. Transcriptome analyses are an essential step for basic and applied research on any organism. Several studies on soybean have focused on the overall and specific transcriptional activity of genes across various tissues, organs, and developmental stages (Cheng et al., 2009; Wong et al., 2009; Fan et al., 2013; 
Wong et al., 2013). Several genes putatively involved in the control of important agronomic traits such as nodule, flower, and seed development have been identified (Wong et al., 2009; Severin et al., 2010; Jung et al., 2012). However, the molecular mechanisms underlying the development of the four-seed pod and the related increase in yield remain poorly understood.

Branching, flowering, and pod formation are three important developmental periods in soybean, and represent the transition from vegetative to reproductive growth. Factors that contribute to the formation of more flowers will result in more pods and more seeds. In this study, we chose the axillary bud (Vegetative6 Stage, V6-Stage), unfertilized ovary (Reproductive Stage, R1-Stage), and young pod (Reproductive Stage, R3-Stage) as the experimental materials. Highthroughput Illumina sequencing was performed for the three different tissues, representing three developmental stages, of the four-seed pod mutant and the control. Based on extensive data analyses, we identified different genes and pathways related to the soybean four-seed mutation. This dataset will serve as the foundation to understand the mechanisms regulating seed and pod development, and will also lay the foundation for the identification of key genes in soybean.

\section{MATERIAL AND METHODS}

\section{Plant materials}

The soybean four-seed pod mutant used in this study was selected by chance through drought-stress experiments at the Plant Biotechnology Center of Jilin Agricultural University. This line was mutated from the soybean variety Jinong 18. Compared with the control, the mutant shows a $20 \%$ higher ratio of four-grain pods. This soybean mutant has been continuously selected since its identification in 2008, and was included in a regional field trial in 2014.

In July 2013, fresh axillary buds (V6-Stage, Figure 1A), unfertilized ovaries (R1-Stage, Figure 1B), and young pods (R3-Stage, Figure 1C) (http://www.soybeanmanagement.info) were collected from the four-grain pod soybean mutant and control growing in the greenhouse of the Plant Biotechnology Center of Jilin Agricultural University. All fresh materials were wrapped in aluminum foil, frozen in liquid nitrogen, and stored at $-80^{\circ} \mathrm{C}$ until analysis.

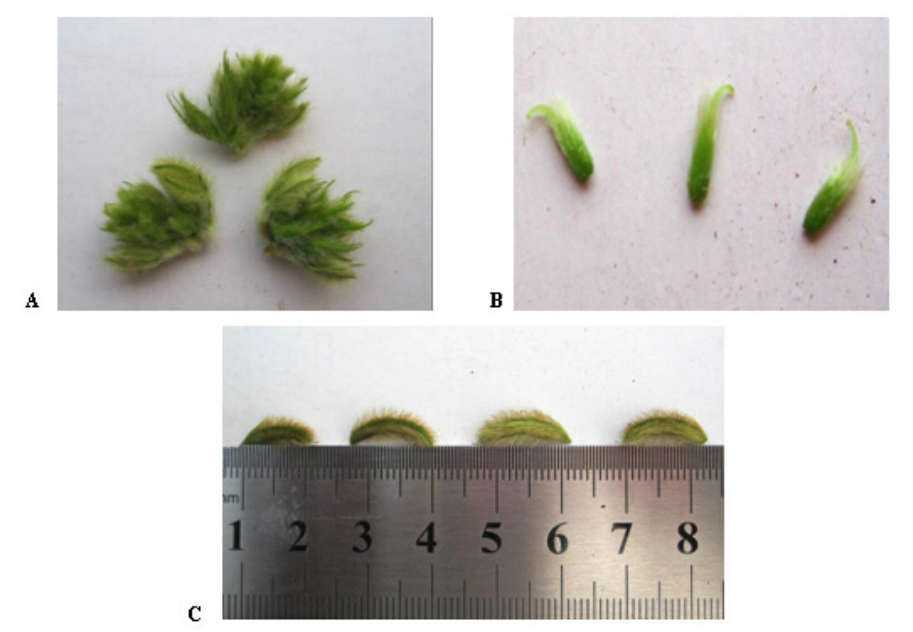

Figure 1. Plant materials in three different periods. A. Fresh axillary buds. B. Unfertilized ovaries. C. Young pods. 


\section{RNA isolation and construction of Illumina sequencing library}

Total RNA was extracted from soybean at the three developmental phases described above using an Eastep ${ }^{\mathrm{TM}}$ Universal RNA Extraction Kit (Promega, Shanghai, China). Oligo (dT) beads were used to isolate poly(A) mRNA from total RNA, according to the Illumina manufacturer instructions. Fragmentation buffer was added to cut the mRNA into short fragments, and the fragments were then used to synthesize first-strand complementary DNA (cDNA) using random hexamer adaptors and reverse transcriptase (Invitrogen, Carlsbad, CA, USA). Second-strand cDNA was synthesized with RNase H (Invitrogen) and DNA polymerase I (NEB). A paired-end library was constructed from the cDNAs synthesized with a Genomic Sample Prep Kit (Illumina). The resulting short fragments with desired lengths were purified with a QIA quick polymerase chain reaction (PCR) (Qiagen, Valencia, CA, USA) Extraction Kit, and then end-repaired and linked to sequencing adapters (Margulies et al. 2005). After the unsuitable fragments were removed with AMPureXP beads, the sequencing library was constructed via PCR amplification. The cDNA library was quantified with Qubit 2.0 fluorescer (Invitrogen) and a cluster of the DNA fragments on the surface of a flow cell chip was amplified using bridge PCR. When the single molecular DNA cluster had been amplified many times, the products were sequenced using the Illumina GAll sequencing platform.

\section{Illumina sequencing and functional annotation}

Quality reads were successively assembled into contigs, scaffolds, and unigenes with Velvet and Oases software packages. We used the method of reads per kilobase of exon model per million mapped reads (RPKM) (Mortazavi et al., 2008) values to normalize the transcript levels. Genes were considered to be differentially expressed when there was a 2-fold difference in their transcript levels between two different tissues. All unigene sequences were used as queries to search the Clusters of Orthologous Groups (COG) database (E-value $\left.<10^{-5}\right)$, and were functionally annotated by Gene Ontology (GO) analysis with Blast2GO software (E-value $<10^{-5}$ ) (http://www. blast2go.com/). Significantly enriched metabolic pathways were predicted by Kyoto Encyclopedia of Genes and Genomes (KEGG) mapping.

\section{Quantitative real-time fluorescent PCR (qRT-PCR) analysis}

To verify the results of transcriptome sequencing obtained from the soybean four-seed pod mutant at different developmental periods, the transcript levels of 10 genes were determined by quantitative real-time fluorescent transcription ( $q R T)-P C R$. According to the sequenced transcriptome data, 10 differentially expressed genes (DEGs) between the control and mutants (log2 ratio $=-19.43-14.36$ ) were selected for analysis. All of these genes had GO functional annotations. For this analysis, the materials used for RNA extraction were the same as those used to construct the digital gene expression (DGE) library. Ten gene-specific primer pairs were designed based on sequences of the target genes using Primer Expression software (Table 1). Each reaction mixture $(25 \mu \mathrm{L})$ contained $2 \mu \mathrm{L}$ cDNA $(25 \mathrm{ng} / \mu \mathrm{L}), 12.5 \mu \mathrm{L} 2 \mathrm{X}$ SYBR premix Ex taq ${ }^{\mathrm{TM}}$ (Takara, Dalian,

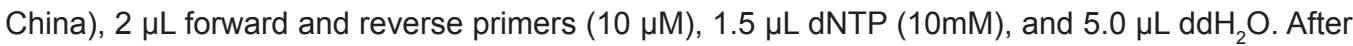
gentle mixing, the PCR mixtures were placed in an Agilent Technologies Stratagene Mx3000P PCR instrument for the thermocycling reaction. The thermal cycling conditions were as follows: initial denaturation at $95^{\circ} \mathrm{C}$ for $3 \mathrm{~min}$, denaturation at $94^{\circ} \mathrm{C}$ for $15 \mathrm{~s}$, annealing at $60^{\circ} \mathrm{C}$, and extension 
for $40 \mathrm{~s}$. The soybean tubulin gene (TUB4) (Fan et al., 2013) was used as the internal control. The relative expression levels of each transcript were calculated using the $2^{-\Delta \Delta C t}$ method (Kenneth and Thomas, 2001): $\left(\Delta \Delta C_{\mathrm{T}}=\left[C_{\mathrm{T} \text {, Target }}-C_{\mathrm{T} \text {, Tub3 } 3}\right]_{\mathrm{Time} \mathrm{x}}-\left[C_{\mathrm{T} \text {, Target }}-C_{\mathrm{T}, \text { Tub3 }}\right)_{\mathrm{Time} 0}\right.$; where time $\mathrm{x}$ is any time point and time 0 represents the $1 \mathrm{X}$ expression of the target gene normalized to that of TUB4. The mean $\mathrm{Ct}$ values for both the target and internal control genes were determined at time zero.

\begin{tabular}{|c|c|c|c|}
\hline No. & $\mathrm{ID}^{\#}$ & Primer sequence $\left(5^{\prime} \rightarrow 3^{\prime}\right)$ & Annotation \\
\hline 1 & Glyma08g22630.1 & $\begin{array}{l}\text { P1: GGAGCCAAATAGAACCGCACATAAC } \\
\text { P2: AGTTCCTGTGTCCGAGTAAGTAGAG }\end{array}$ & Protein folding \\
\hline 2 & Glyma16g01440.1 & $\begin{array}{l}\text { P1: GGATGTTCCTGGTCTCTCCAAGTCT } \\
\text { P2: GAATCCAACСTCTCСTCTCСАACCT }\end{array}$ & Unfolded protein binding \\
\hline 3 & Glyma15g41880.1 & $\begin{array}{l}\text { P1: CCCACATACCCATCTACCCAAAGC } \\
\text { P2: TCTCTGAACCTCTCCGTCCTGAC }\end{array}$ & Protein folding \\
\hline 4 & Glyma09g04500.1 & $\begin{array}{l}\text { P1: AGGTCAGTGAGGAGGTTGCCAAGTA } \\
\text { P2: AGAAGGTCCACGAGTTGTAAGGATGG }\end{array}$ & Unknown \\
\hline 5 & Glyma20g28360.1 & $\begin{array}{l}\text { P1: ACACAACTCTTTAAGGATGGCAACCC } \\
\text { P2: AAGATACAAGACACAAGCCGAGTACG }\end{array}$ & Copper ion binding \\
\hline 6 & Glyma02g03280.1 & $\begin{array}{l}\text { P1: GACTGCTTGCTTCAATGC } \\
\text { P2: CGATGCTGCTATGTCTCA }\end{array}$ & Peptidase activity \\
\hline 7 & Glyma08g11400.1 & $\begin{array}{l}\text { P1: ATCTTGAGTGGTATCTGGAA } \\
\text { P2: TCTGTTCTTCAATTCTACCG }\end{array}$ & Protein binding \\
\hline 8 & Glyma08g44950.1 & $\begin{array}{l}\text { P1: GGACTGGTGGAGGAACAATA } \\
\text { P2: AAGTTGACACCGTAGCCT }\end{array}$ & DNA binding \\
\hline 9 & Glyma08g45840.2 & $\begin{array}{l}\text { P1: CCCTGTGATGACCAAGAA } \\
\text { P2: АACCTCAACAGAATCCATCC }\end{array}$ & Unknown \\
\hline 10 & Glyma07g00260.1 & $\begin{array}{l}\text { P1: AGACTGCTTGCTTCAATG } \\
\text { P2: CTTTCAGTGCTTTCTCCATT }\end{array}$ & Response to red or far red light \\
\hline TUB4 & EV263740 & $\begin{array}{l}\text { P1: GGCGTCCACATTCATTGGA } \\
\text { P2: CCGGTGTACCAATGCAAGAA }\end{array}$ & Beta-tubulin \\
\hline
\end{tabular}

\#ID is the ID of each differential gene. Annotation refers to the functional annotation of each gene in the GO database.

\section{RESULTS}

\section{Illumina sequence data and assembly}

The cDNAs obtained from the four-grain pod soybean mutant (JN18MT01) and the control (JN18) at three growth phases (axillary bud, young pod, and unfertilized ovary) were sequenced using Illumina sequencing technology. Large transcriptome datasets were generated for each sample. The smallest dataset was obtained from the young-pod mutant sample $(4,017,760,362$ bp) and the largest was from the unfertilized ovary control sample $(4,686,416,658 \mathrm{bp})$. In each sample, $100 \%$ of the clean reads had quality scores at the cycleQ20 level, and the GC content was approximately $46-48 \%$ (Table 2 ). When sequence data were mapped against the soybean reference genome (allowing a $\leq 2$-bp mismatch for each read), there was an average of $67.13 \%$ mapped reads, $44.13 \%$ perfect reads, and $96.89 \%$ InDels (Table 2 ).

\section{Screening to detect differentially expressed genes}

The strategies used to detect DEGs, the pairs of samples compared, and the number of DEGs between each pair of samples are shown in Table 3. In total, 4183 DEGs were detected in the mutant and the source variety. The highest number of DEGs was found between the axillary 
bud mutant and the source variety (2138 up-regulated and 41 down-regulated genes). The fewest number of DEGs was found between the young pod mutant and the control (69 up-regulated and 22 down-regulated genes) (Table 3). Among them, the log2 ratio value of 13 DEGs was greater than 10. The DEGs in the three different growth phases of soybean and the overlaps among them are illustrated in a Venn diagram (Figure 2). This diagram shows that 13 DEGs were expressed in the axillary bud, young pod, and unfertilized ovary; 11 of these were up-regulated and two were down- regulated (Table 4). These genes were further evaluated to investigate their biological functions. The scatter plot showed that the expression of up- and down-regulated genes in the mutants and the control fitted a normal distribution at the V6-Stage (Figure 3A), R1- Stage (Figure 3C), and R3-Stage (Figure 3B).

Table 2. Summary of raw reads and reference statistics for six samples.

\begin{tabular}{lcccccc}
\hline Sample & Data (bp) & GC (\%) & Cycle Q20 (\%) & Mapped reads & Perfect reads & InDel \\
\hline Axillary bud mutant & $4,180,012,452$ & 46.73 & 100 & $14,050,443 / 66.55 \%$ & $6,426,153 / 45.73 \%$ & $13,618,761 / 96.92 \%$ \\
Axillary bud control & $4,408,624,836$ & 48.21 & 100 & $15,045,492 / 67.57 \%$ & $5,314,605 / 35.32 \%$ & $14,559,026 / 96.76 \%$ \\
Young pod mutant & $4,017,760,362$ & 46.26 & 100 & $13,601,515 / 67.02 \%$ & $6,168,703 / 45.35 \%$ & $13,176,640 / 96.87 \%$ \\
Young pod control & $4,121,206,254$ & 46.72 & 100 & $13,598,653 / 65.33 \%$ & $6,211,103 / 45.67 \%$ & $13,181,916 / 96.93 \%$ \\
Ovary mutant & $4,400,207,856$ & 46.48 & 100 & $15,186,073 / 68.33 \%$ & $7,018,883 / 46.21 \%$ & $14,712,213 / 96.87 \%$ \\
Ovary control & $4,686,416,658$ & 46.34 & 100 & $16,094,490 / 67.99 \%$ & $7,487,011 / 46.51 \%$ & $15,605,648 / 96.96 \%$ \\
\hline
\end{tabular}

Data are total nucleotides. GC (\%) is the proportion of guanidine and cytosine nucleotides among the total nucleotides. CycleQ20 (\%) is the proportion of nucleotides with a quality value larger than 20. Mapped Reads represents the number of reads and comparison with reference genome alignment. Perfect Reads represents no mismatch, no insertion deletion, the only reads the comparison on the genome. InDel represents insertion deletion reads.

Table 3. Statistical analysis of differentially expressed genes between the mutant and the source variety.

\begin{tabular}{lccc}
\hline Type & ABC1_vs_ABM2 & OVC1_vs_OVM2 & YPC1_vs_YPM2 \\
\hline Total number of DEGs & 2179 & 2060 & 91 \\
Number of upregulated genes & 2138 & 1381 & 69 \\
Number of downregulated genes & 41 & 679 & 22 \\
Number of $\mid$ log2 ration| $\geq 10$ & 74 & 75 & 13
\end{tabular}

ABC1 = axillary bud control; $\mathrm{ABM}$ = axillary bud mutant; YPC1 = young pod control; YPM2 = young pod mutant; OVC1 = unfertilized ovary control; OVM2 = unfertilized ovary mutant.

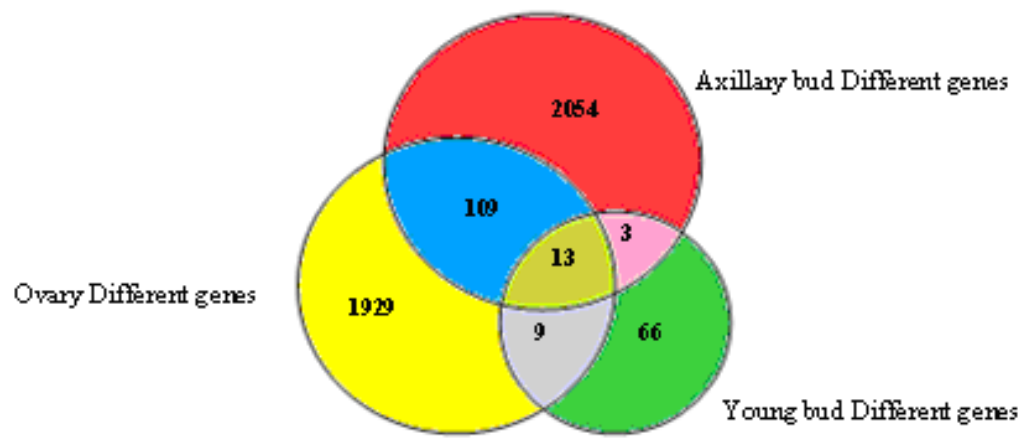

Figure 2. Venn diagram showing overlaps among differentially expressed genes of the soybean mutant and control in three different periods. Numbers outside the circles denote the total number of differentially expressed genes in three different periods. Numbers in one circle denote differentially expressed genes, and numbers in two or more intersecting circles denote overlapping genes. 


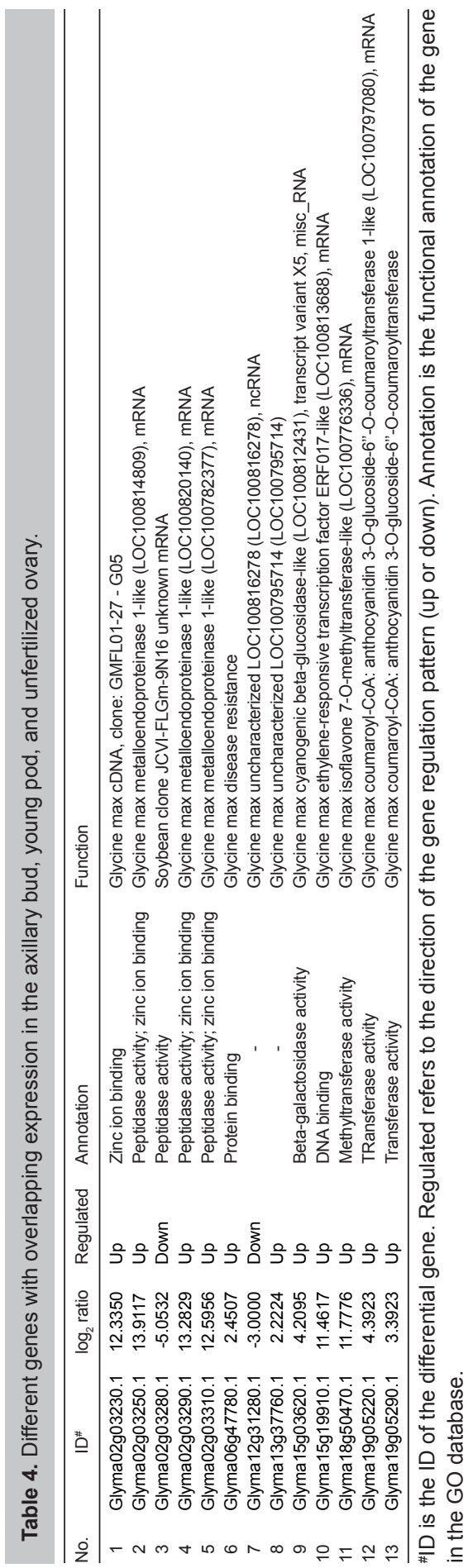



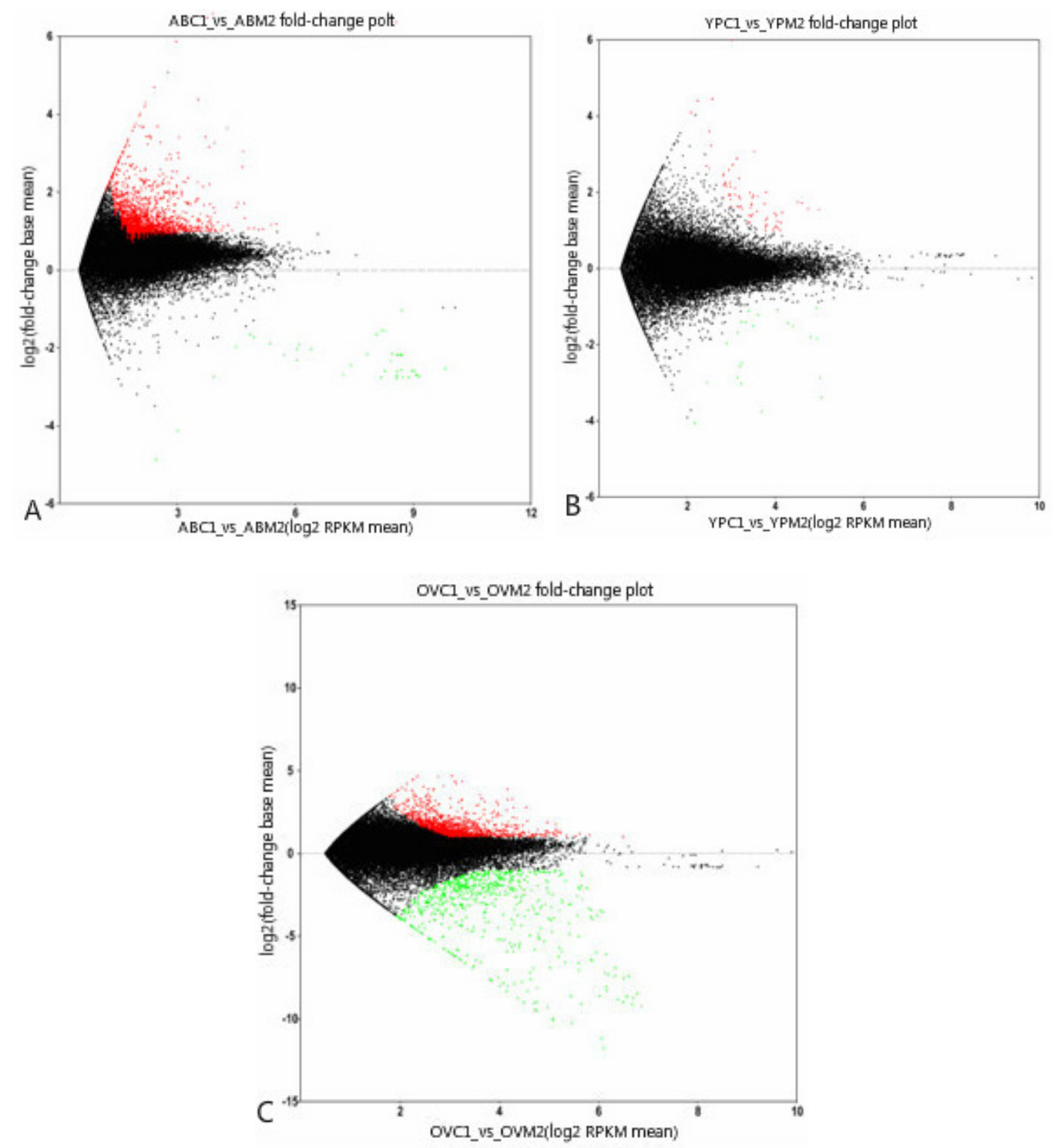

Figure 3. Scatter plot of soybean control and mutant differentially expressed genes in three different periods. A. Axillary bud control and mutant. B. Young pod control and mutant. C. Ovary control and mutant. Red represents upregulation genes, green represents downregulated genes. Abscissa represents the relative log2 RPKM mean, ordinate represented fold change base mean.

\section{Functional annotation of differentially expressed genes}

The assembled unigenes were further analyzed to determine their pathway annotations (KEGG), COG functional annotations, and GO functional annotations. 


\section{Gene Ontology functional analysis}

GO is an internationally standardized gene function classification system. It provides dynamically updated and controlled vocabulary to fully describe the attributes of genes and gene products in an organism. The GO database is suitable to analyze genes from various species, and it can limit and describe genes and proteins. In GO analyses, genes are classified into three main categories; biological process, cellular component, and molecular function. The DEGs in the axillary bud (Figure 4A), young pod (Figure 4B), and unfertilized ovary (Figure 4C) were further classified into 18 subcategories in the cellular component category, 18 subcategories in the molecular function category, and 25 subcategories in the biological process category.

The percentage of DEGs in each category was similar across the three different growth phases. Of the 583,516 unigenes with known functions, 208,516 were assigned to the molecular function category, 74,563 were assigned to the cellular component category, and 300,437 were assigned to the biological process category. However, when the DEGs were compared among the three different developmental stages, there were clear differences in the distribution of subcategories. In the cellular component category, the cell part subcategory had the largest number of DEGs ( 65 or $28.26 \%$ of DEGs in the axillary bud, 1737 or $23.22 \%$ of DEGs in the young pod, and 1759 or $21.5 \%$ of DEGs in the unfertilized ovary). In the biological process category, DEGs in the cellular process and metabolic process subcategories were the most abundant (1563 or $13.61 \%$ of DEGs in the axillary bud, 54 or $13.37 \%$ of DEGs in the young pod, and 1557 or $12.42 \%$ of DEGs in the unfertilized ovary). In the molecular function category, DEGs in the binding protein subcategory were the most abundant (1254 or $42.95 \%$ of DEGs in the axillary bud, 42 or $43.3 \%$ of DEGs in the young pod, and 1303 or $44.62 \%$ of DEGs in the unfertilized ovary).

\section{COG functional annotation}

The orthologous classification of genetic products was conducted using tools at the COG database (COG, http://www.ncbi.nlm.nih.gov/COG/). It is assumed that each COG protein evolved from a specific ancestor (Tatusov et al., 2003). The COG database contains protein sequences encoded in the genomes of algae, bacteria, and eukaryotes, and provides information about their evolutionary relationships (Lulin et al., 2012). DEGs between the soybean mutant and the control at the three different growth periods were further annotated based on COG categories. The DEGs were assigned COG functional annotations, which could be grouped into 25 different functional categories. In the soybean axillary bud, the largest category of DEGs was general function prediction only $(231,19.66 \%)$, followed by transcription $(93,7.91 \%)$, carbohydrate transport and metabolism $(91,7.74 \%)$, posttranslational modification, protein turnover, chaperones $(81,6.89 \%)$, signal transduction mechanisms $(79,6.72 \%)$, amino acid transport and metabolism $(73,6.21 \%)$, energy production and conversion $(57,4.85 \%)$, secondary metabolites biosynthesis, transport, and catabolism $(57,4.85 \%)$, replication, recombination, and repair $(54,4.60 \%)$, function unknown (57, 4.85\%), and others (Figure 5A). In the young pod, the largest category of DEGs was general function prediction only $(11,26.83 \%)$, followed by carbohydrate transport and metabolism $(5$, $12.20 \%$ ), secondary metabolites biosynthesis, transport, and catabolism $(5,12.20 \%)$, amino acid transport and metabolism $(3,7.31 \%)$, posttranslational modification, protein turnover, chaperones $(2,4.88 \%)$, signal transduction mechanisms $(2,4.88 \%)$, function unknown $(2,4.88 \%)$, and others (Figure 5B). In the unfertilized ovary, the largest category of DEGs was general function prediction only $(209,16.95 \%)$, followed by posttranslational modification, protein turnover, chaperones 

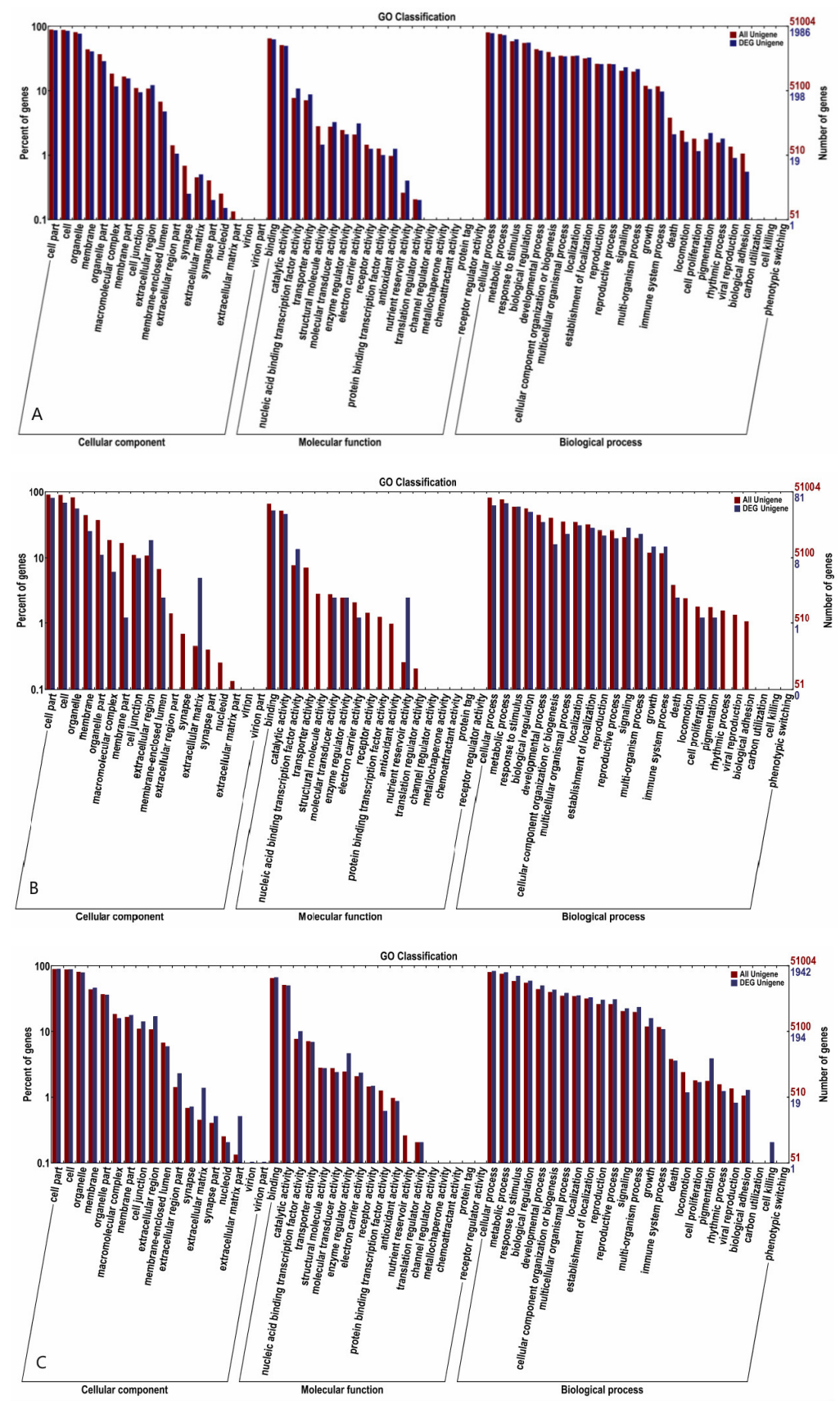

Figure 4. GO functional categorization of significantly differentially expressed genes in three different periods. Soybean axillary bud control and mutant (A), soybean young pod control and mutant (B), soybean ovary control and mutant (C). Abscissa represented gene function classification by GO analysis, from left to right in turn = Cellular component, Molecular function, Biological process. Left ordinate represents the percentage of genes, right ordinate represents the number of genes. In addition, the red column represents all unigenes and the blue column represents DEG unigenes. 
(183, 14.84\%), transcription (105, 8.52\%), carbohydrate transport and metabolism $(85,6.89 \%)$, signal transduction mechanisms $(83,6.73 \%)$, replication, recombination, and repair $(78,6.33 \%)$, translation, ribosomal structure, and biogenesis $(77,6.25 \%)$, amino acid transport and metabolism $(69,5.60 \%)$, secondary metabolites biosynthesis, transport, and catabolism $(61,4.95 \%)$, function unknown (40, 3.24\%), and others (Figure 5C).
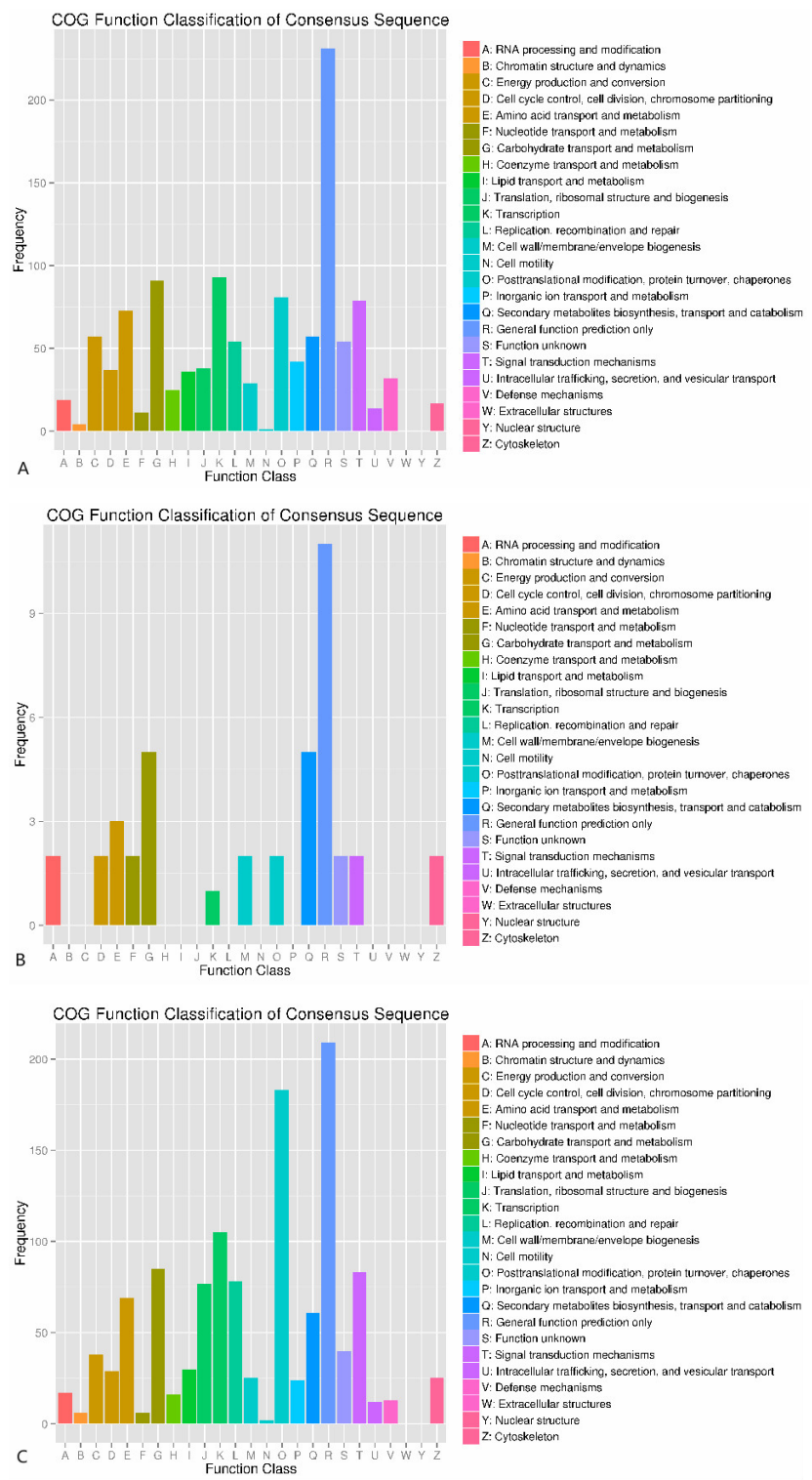

Figure 5. COG function classification of consensus sequences in three different periods. Soybean axillary bud contrast and mutant (A), soybean young pod contrast and mutant (B), soybean ovary contrast and mutant (C). Abscissa represents gene function classification by COG analysis, including 25 different functions; different capital letters represent different functions; ordinates represents gene frequency. Different colored columns represent different gene functions. 


\section{Significant enrichment analysis of KEGG pathways}

Within an organism, different genes coordinate to perform biological functions. Analyses based on pathways can provide information about the biological functions of genes. Gene functions were analyzed using the hypergeometric model in KEGG to identify pathways significantly enriched with DEGs. These analyses revealed the main signal transduction pathways and biochemical metabolic pathways in which the DEGs were involved.

In this study, pathways with a $Q_{\text {_value of }} \leq 0.05$ were defined as those that were significantly enriched with DEGs. The sequencing results showed that a total of 646 unigenes of DEGs were involved in 103 metabolic pathways. At the axillary bud growth phase, a total of 305 unigenes of DEGs were involved in 93 metabolic pathways. At the young pod growth stage, three unigenes of DEGs were involved in four pathways. At the unfertilized ovary growth stage, 338 unigenes of DEGs were involved in 81 metabolic pathways. Among these metabolic pathways, eight had Q_values of $\leq 0.05$ (Table 5). These eight pathways consisted of those associated with amino acid metabolism (beta-alanine metabolism, arginine and proline metabolism, and amino sugar and nucleotide sugar metabolism), those related to carbon metabolism (starch and sucrose metabolism, and glyoxylate and dicarboxylate metabolism), and those related to plant hormone signal transduction and protein processing in the endoplasmic reticulum. Metabolic maps were constructed after aligning DEGs to the KEGG database.

\begin{tabular}{|c|c|c|c|c|c|c|}
\hline No. & \#KEGG_Pathway & ko_ID & Cluster_frequency & Genome_frequency & $P$ value & Q_value \\
\hline 1 & Plant hormone signal transduction & ko04075 & 57 of $305 / 18.69 \%$ & 742 of $9217 / 8.05 \%$ & 1.04E-09 & $9.66 \mathrm{E}-08$ \\
\hline 2 & Glyoxylate and dicarboxylate metabolism & ko00630 & 15 of $305 / 4.92 \%$ & 96 of $9217 / 1.04 \%$ & $5.15 \mathrm{E}-07$ & 4.79E-05 \\
\hline 3 & Beta-Alanine metabolism & ko00410 & 11 of $305 / 3.61 \%$ & 101 of $9217 / 1.10 \%$ & 0.000487 & 0.045289 \\
\hline 4 & Arginine and proline metabolism & ko00330 & 17 of $305 / 5.57 \%$ & 208 of $9217 / 2.26 \%$ & 0.000528 & 0.049122 \\
\hline 5 & Amino sugar and nucleotide sugar metabolism & ko00520 & 2 of $3 / 66.67 \%$ & 249 of $9217 / 2.70 \%$ & 0.002142 & 0.008568 \\
\hline 6 & Starch and sucrose metabolism & ko00500 & 2 of $3 / 66.67 \%$ & 296 of $9217 / 3.21 \%$ & 0.003018 & 0.012073 \\
\hline 7 & Valine, leucine, and isoleucine biosynthesis & ko00290 & 1 of $3 / 33.33 \%$ & 90 of $9217 / 0.98 \%$ & 0.029012 & 0.116047 \\
\hline 8 & Protein processing in the endoplasmic reticulum & ko04141 & 52 of $338 / 15.38 \%$ & 429 of $9217 / 4.66 \%$ & $2.01 \mathrm{E}-12$ & $1.63 \mathrm{E}-10$ \\
\hline
\end{tabular}

\section{Cluster analysis of differentially expressed genes based on expression patterns}

We conducted a cluster analysis of DEGs based on their expression patterns; that is, those genes with the same or similar expression patterns were grouped together. Such analyses can provide important clues to the roles of functionally unknown genes in biological processes, because genes sharing the same expression pattern have related functions. Cluster analysis of DEGs between the soybean mutant and the control was conducted through average linkage distance, i.e., genes with similar functions were clustered together in the taxonomic tree. In this way, genes with a known function can shed light on the functions of unknown genes. The results of the cluster analyses are summarized in Table 6 and Figure 6 with the young pod mutant and control as an example.

\section{Validation of transcriptome data by qRT-PCR}

We conducted quantitative real-time polymerase chain reaction (qRT-PCR) analyses to verify the Solexa sequencing results. Specific primers used in the qRT-PCRs were designed for 
10 candidate genes using Beacon Designer 7.5 SYBR ${ }^{\circledR}$ Green Design, and TUB4 served as the internal control. Reverse transcription was conducted using the same RNA samples as were used in the Solexa sequencing, and internal control and candidate genes were amplified at the same time. The qRT-PCR results of the expression patterns of candidate genes were generally consistent with the Solexa sequencing results (Figure 7), confirming the reliability of the transcriptome data. The significant differences in transcript abundance may result from the different algorithms used in the two technologies.

\begin{tabular}{|c|c|c|c|c|c|c|c|c|}
\hline No. & $\mathrm{ID}^{\#}$ & YPM & YPC & Chi & Q_value & log2 ratio & Regulated & Annotation \\
\hline 1 & Glyma02g03250.1 & 96 & 1003 & 0 & 0 & -3.3851 & Down & Metalloendopeptidase activity \\
\hline 2 & Glyma02g03290.1 & 125 & 918 & 0 & 0 & -2.8766 & Down & Metalloendopeptidase activity \\
\hline 3 & Glyma02g03230.1 & 175 & 612 & 0 & 0 & -1.8062 & Down & Metalloendopeptidase activity \\
\hline 4 & Glyma02g03310.1 & 212 & 755 & 0 & 0 & -1.8324 & Down & Metalloendopeptidase activity \\
\hline 5 & Glyma02g13290.1 & 125 & 918 & 0 & 0 & -2.8766 & Down & Metalloendopeptidase activity \\
\hline 6 & Glyma06g08860.1 & 116 & 335 & 0 & 0 & -1.5300 & Down & Catalytic activity \\
\hline 7 & Glyma04g08760.1 & 103 & 281 & 0 & 0 & -1.4479 & Down & Nucleotide transport and metabolism \\
\hline 8 & Glyma14g06990.1 & 11 & 155 & 0 & 0 & -3.8167 & Down & Serine-type endopeptidase activity \\
\hline 9 & Glyma06g45050.1 & 540 & 181 & 0 & 0 & 1.5770 & Up & O-methyltransferase activity \\
\hline 10 & Glyma12g12230.1 & 397 & 118 & 0 & 0 & 1.7504 & Up & O-methyltransferase activity \\
\hline 11 & Glyma12g31280.1 & 454 & 137 & 0 & 0 & 1.7285 & Up & Unknown \\
\hline 12 & Glyma14g02240.1 & 752 & 258 & 0 & 0 & 1.5434 & Up & Lipid transport \\
\hline 13 & Glyma02g44200.1 & 116 & 31 & 0 & 0 & 1.9038 & Up & Plasma membrane \\
\hline 14 & Glyma12g26780.1 & 142 & 56 & 0 & 0 & 1.3424 & Up & Sequence-specific DNA binding transcription factor activity \\
\hline 15 & Glyma17g07740.1 & 169 & 80 & 0 & 0 & 1.0790 & Up & Nucleotide binding \\
\hline 16 & Glyma02g37020.1 & 177 & 82 & 0 & 0 & 1.1101 & Up & Nucleotide binding \\
\hline 17 & Glyma10g36910.1 & 141 & 43 & 0 & 0 & 1.7133 & Up & Response to stress \\
\hline 18 & Glyma15g13750.1 & 188 & 90 & 0 & 0 & 1.0627 & Up & Unknown \\
\hline 19 & Glyma13g39890.1 & 117 & 14 & 0 & 0 & 3.0630 & Up & Cytoplasmic membrane-bounded vesicle \\
\hline 20 & Glyma16g28150.1 & 201 & 100 & 0 & 0 & 1.0072 & Up & Response to stress \\
\hline 21 & Glyma06g45380.1 & 212 & 91 & 0 & 0 & 1.2201 & Up & Unknown \\
\hline 22 & Glyma09g10340.1 & 185 & 67 & 0 & 0 & 1.4653 & Up & Very long-chain fatty acid metabolic process \\
\hline 23 & Glyma17g03350.1 & 191 & 73 & 0 & 0 & 1.3876 & Up & Response to biotic stimulus \\
\hline 24 & Glyma14g04580.1 & 154 & 38 & 0 & 0 & 2.0189 & Up & Plasmodesma \\
\hline 25 & Glyma15g13770.1 & 191 & 81 & 0 & 0 & 1.2376 & Up & Unknown \\
\hline 26 & Glyma15g13760.1 & 233 & 83 & 0 & 0 & 1.4891 & Up & Unknown \\
\hline 27 & Glyma11g14580.1 & 68 & 13 & 0 & 0 & 2.3870 & Up & Ubiquitin-protein ligase activity \\
\hline 28 & Glyma05g02950.1 & 89 & 29 & 0 & 0 & 1.6178 & Up & Carboxylesterase activity \\
\hline 29 & Glyma15g19910.1 & 67 & 9 & 0 & 0 & 2.8962 & Up & DNA binding \\
\hline 30 & Glyma12g35550.1 & 91 & 34 & 0.000001 & 0.000232 & 1.4203 & Up & DNA binding \\
\hline 31 & Glyma02g03280.1 & 63 & 0 & 0 & 0 & 15.9431 & Up & Peptidase activity \\
\hline 32 & Glyma12g09830.1 & 92 & 29 & 0 & 0 & 1.6656 & Up & Nucleic acid binding \\
\hline 33 & Glyma06g45370.1 & 125 & 62 & 0.00001 & 0.001872 & 1.0116 & Up & Unknown \\
\hline 34 & Glyma20g30700.1 & 92 & 28 & 0 & 0 & 1.7162 & Up & Response to stress \\
\hline 35 & Glyma05g35170.1 & 105 & 37 & 0 & 0 & 1.5048 & Up & Unknown \\
\hline 36 & Glyma19g29880.1 & 91 & 23 & 0 & 0 & 1.9842 & Up & 2-isopropylmalate synthase activity \\
\hline 37 & Glyma15g10710.1 & 127 & 60 & 0.000003 & 0.000633 & 1.0818 & Up & Unknown \\
\hline 38 & Glyma12g07190.1 & 133 & 60 & 0 & 0 & 1.1484 & Up & Monooxygenase activity \\
\hline 39 & Glyma17g15460.1 & 100 & 26 & 0 & 0 & 1.9434 & Up & DNA binding \\
\hline 40 & Glyma11g18460.1 & 98 & 27 & 0 & 0 & 1.8598 & Up & Nucleic acid binding \\
\hline 41 & Glyma08g48040.1 & 131 & 60 & 0.000001 & 0.000232 & 1.1265 & Up & Regulation of transcription, DNA-dependent \\
\hline 42 & Glyma17g03360.1 & 134 & 55 & 0 & 0 & 1.2847 & Up & Response to biotic stimulus \\
\hline 43 & Glyma18g53440.1 & 159 & 79 & 0.000001 & 0.000232 & 1.0091 & Up & Regulation of transcription, DNA-dependent \\
\hline 44 & Glyma13g35950.1 & 64 & 17 & 0 & 0 & 1.9125 & Up & Copper ion binding \\
\hline 45 & Glyma13g37760.1 & 66 & 18 & 0 & 0 & 1.8745 & Up & Unknown \\
\hline 46 & Glyma14g04300.1 & 58 & 8 & 0 & 0 & 2.8580 & Up & Cell part \\
\hline 47 & Glyma06g45410.1 & 81 & 31 & 0.000005 & 0.000986 & 1.3857 & Up & Unknown \\
\hline 48 & Glyma07g37270.1 & 85 & 33 & 0.000004 & 0.000815 & 1.3650 & Up & Metal ion binding \\
\hline 49 & Glyma09g01610.1 & 50 & 10 & 0 & 0 & 2.3219 & Up & Protein binding \\
\hline 50 & Glyma06g35710.1 & 58 & 18 & 0.000008 & 0.001541 & 1.6881 & Up & Sequence-specific DNA binding transcription factor activity \\
\hline
\end{tabular}

Continued on next page 


\begin{tabular}{|c|c|c|c|c|c|c|c|c|}
\hline No. & $\mathrm{ID}^{\#}$ & YPM & YPC & Chi & Q_value & $\log 2$ ratio & Regulated & Annotation \\
\hline 51 & Glyma12g06460.1 & 50 & 11 & 0.000001 & 0.000232 & 2.1844 & Up & Ubiquitin-protein ligase activity \\
\hline 52 & Glyma13g35850.1 & 49 & 12 & 0.000004 & 0.000815 & 2.0297 & Up & Copper ion binding \\
\hline 53 & Glyma09g04750.1 & 58 & 13 & 0 & 0 & 2.1575 & Up & Ubiquitin-protein ligase activity \\
\hline 54 & Glyma20g38140.1 & 79 & 34 & 0.000045 & 0.007421 & 1.2163 & Up & Protein binding \\
\hline 55 & Glyma02g09000.1 & 67 & 22 & 0.000004 & 0.000815 & 1.6067 & Up & Response to mechanical stimulus \\
\hline 56 & Glyma07g31300.1 & 65 & 21 & 0.000004 & 0.000815 & 1.6301 & Up & Copper ion binding \\
\hline 57 & Glyma09g08330.1 & 50 & 8 & 0 & 0 & 2.6439 & Up & DNA binding \\
\hline 58 & Glyma07g05620.1 & 61 & 19 & 0.000005 & 0.000986 & 1.6828 & Up & DNA binding \\
\hline 59 & Glyma04g06720.1 & 57 & 14 & 0.000001 & 0.000232 & 2.0255 & Up & Unknown \\
\hline 60 & Glyma13g01930.1 & 75 & 32 & 0.000061 & 0.009565 & 1.2288 & Up & DNA binding \\
\hline 61 & Glyma10g32830.1 & 33 & 1 & 0 & 0 & 5.0444 & Up & Serine-type endopeptidase inhibitor activity \\
\hline 62 & Glyma11g03900.1 & 41 & 8 & 0.000004 & 0.000815 & 2.3576 & Up & DNA binding \\
\hline 63 & Glyma16g29670.1 & 48 & 15 & 0.000052 & 0.008332 & 1.6781 & Up & Response to biotic stimulus \\
\hline 64 & Glyma19g01910.1 & 42 & 7 & 0.000001 & 0.000232 & 2.5850 & Up & Transferase activity, transferring hexosyl groups \\
\hline 65 & Glyma06g10700.1 & 44 & 9 & 0.000003 & 0.000633 & 2.2895 & Up & Plant-type cell wall \\
\hline 66 & Glyma08g16810.1 & 48 & 13 & 0.000012 & 0.002218 & 1.8845 & Up & Ethylene biosynthetic process \\
\hline 67 & Glyma13g04780.1 & 41 & 7 & 0.000002 & 0.000438 & 2.5502 & Up & Transferase activity, transferring hexosyl groups \\
\hline 68 & Glyma08g45600.1 & 43 & 9 & 0.000004 & 0.000815 & 2.2563 & Up & Endopeptidase inhibitor activity \\
\hline 69 & Glyma13g35820.1 & 46 & 12 & 0.000013 & 0.002385 & 1.9386 & Up & Unknown \\
\hline 70 & Glyma17g12710.1 & 43 & 9 & 0.000004 & 0.000815 & 2.2563 & Up & Unknown \\
\hline 71 & Glyma13g17250.1 & 29 & 2 & 0.000002 & 0.000438 & 3.8580 & Up & Sequence-specific DNA binding transcription factor activity \\
\hline 72 & Glyma17g38010.1 & 30 & 3 & 0.000004 & 0.000815 & 3.3219 & Up & Nucleic acid binding \\
\hline 73 & Glyma12g34580.1 & 39 & 10 & 0.000053 & 0.008455 & 1.9635 & Up & Copper ion binding \\
\hline 74 & Glyma11g25660.1 & 21 & 0 & 0.000006 & 0.001168 & 14.3581 & Up & Calcium ion binding \\
\hline 75 & Glyma07g30520.1 & 25 & 3 & 0.000045 & 0.007421 & 3.0589 & Up & Detection of biotic stimulus \\
\hline 76 & Glyma04g10870.1 & 27 & 4 & 0.000051 & 0.008207 & 2.7549 & Up & Plant-type cell wall \\
\hline 77 & Glyma11g25670.1 & 17 & 0 & 0.000048 & 0.00781 & 14.0533 & Up & Calcium ion binding \\
\hline 78 & Glyma07g13790.1 & 88 & 186 & 0 & 0 & -1.0797 & Down & Hydrolase activity \\
\hline 79 & Glyma08g13440.1 & 9 & 77 & 0 & 0 & -3.0969 & Down & Nutrient reservoir activity \\
\hline 80 & Glyma03g28850.1 & 33 & 94 & 0 & 0 & -1.5102 & Down & Glucan endo-1,3-beta-D-glucosidase activity \\
\hline 81 & Glyma19g05220.1 & 13 & 75 & 0 & 0 & -2.5284 & Down & Transferase activity \\
\hline 82 & Glyma07g13780.1 & 44 & 103 & 0 & 0 & -1.2271 & Down & Hydrolase activity \\
\hline 83 & Glyma14g06980.1 & 9 & 68 & 0 & 0 & -2.9175 & Down & Protein binding \\
\hline 84 & Glyma19g05290.1 & 12 & 72 & 0 & 0 & -2.5850 & Down & O-acyltransferase activity \\
\hline 85 & Glyma07g13800.1 & 44 & 103 & 0 & 0 & -1.2271 & Down & Hydrolase activity \\
\hline 86 & Glyma17g07690.1 & 18 & 59 & 0.000002 & 0.000438 & -1.7127 & Down & Amino acid transport \\
\hline 87 & Glyma18g50470.1 & 34 & 75 & 0.000046 & 0.007535 & -1.1414 & Down & 8-hydroxyquercitin 8-O-methyltransferase activity \\
\hline 88 & Glyma13g01570.2 & 24 & 63 & 0.000016 & 0.002871 & -1.3923 & Down & Response to abiotic stimulus \\
\hline 89 & Glyma06g47780.1 & 11 & 44 & 0.000005 & 0.000986 & -2.0000 & Down & Protein kinase activity \\
\hline 90 & Glyma05g30300.1 & 3 & 26 & 0.000014 & 0.002549 & -3.1155 & Down & Nutrient reservoir activity \\
\hline 91 & Glyma15g03620.1 & 1 & 19 & 0.000043 & 0.007171 & -4.2479 & Down & Glucan exo-1,3-beta-glucosi -dase activity \\
\hline
\end{tabular}

\#ID is the ID of each differential gene. YPM is the young pod mutant. YPC is the young pod contrast. Chi is the P value obtained by the Chi-square test. $Q_{-}$value is the $P$ value of the Chi-square test after FDR correction. $\log 2(A / B)$ is the log2 value of two samples ratio. Regulated refers to the direction of the gene regulation pattern (up or down). Annotation is the functional annotation of the gene in the GO database.

\section{DISCUSSION}

Illumina sequencing technology has become an indispensable tool in genomics, and has been used in a diverse range of biological studies. Comparisons between the transcriptomes of the soybean four-seed pod mutant and the control plant at three developmental stages have laid a foundation for the understanding of changes in gene expression during different development phases. We obtained a large dataset ( $25.81 \mathrm{Gbp}$ raw data) by lllumina sequencing, and 55,582 expressed genes were identified by comparison with the soybean reference genome. The sequencing depth and assembly efficiency in this study were greater than those reported previously (Fan et al., 2013). 


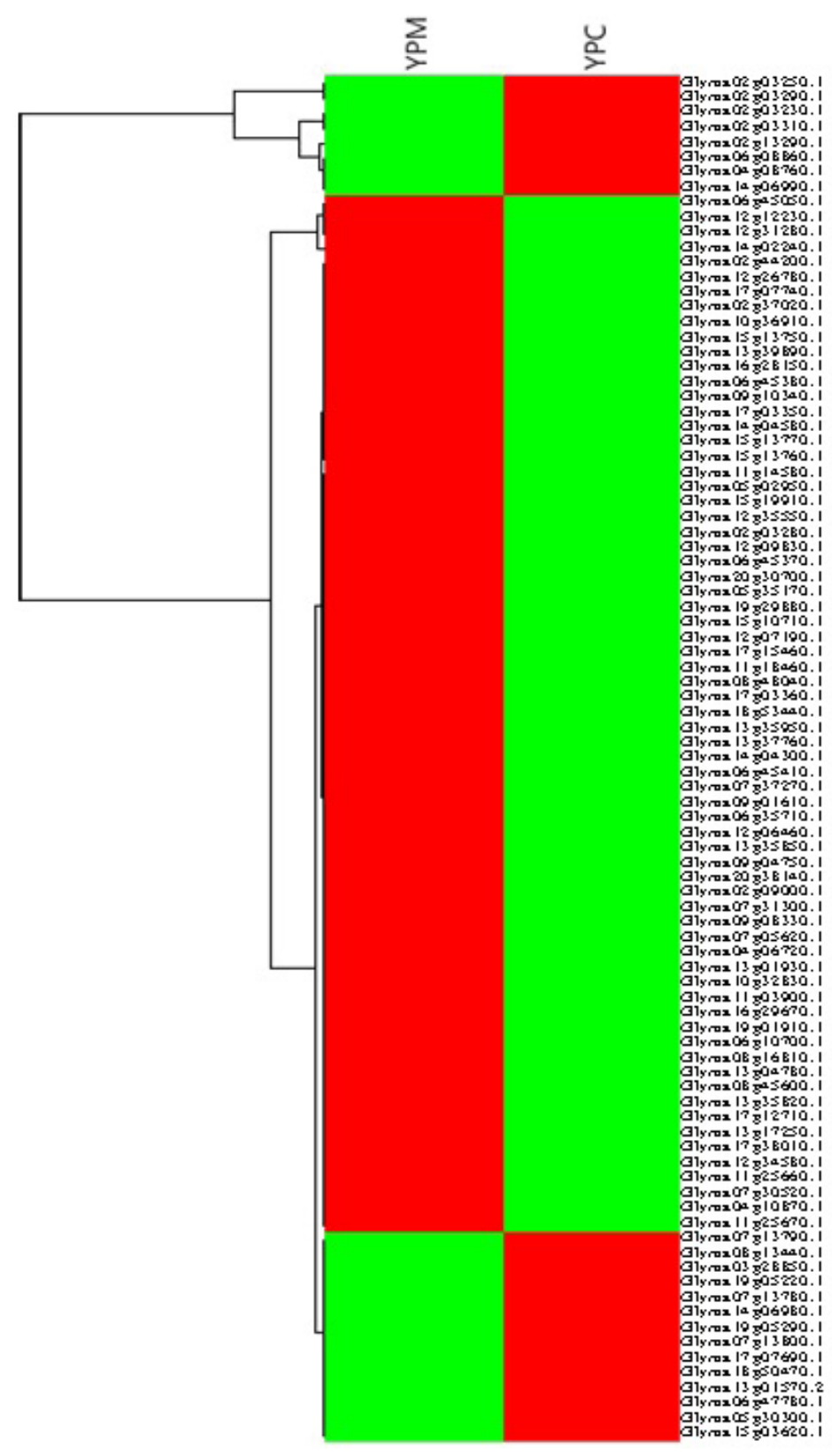

ำ ำ ㅇำ ㅇํㄱ

Figure 6. Differential gene expression pattern clustering analysis in soybean young pod mutant and control. Clustering figure columns represent two samples; lines represented different genes. Red represents high gene expression; green represents lower gene expression. 


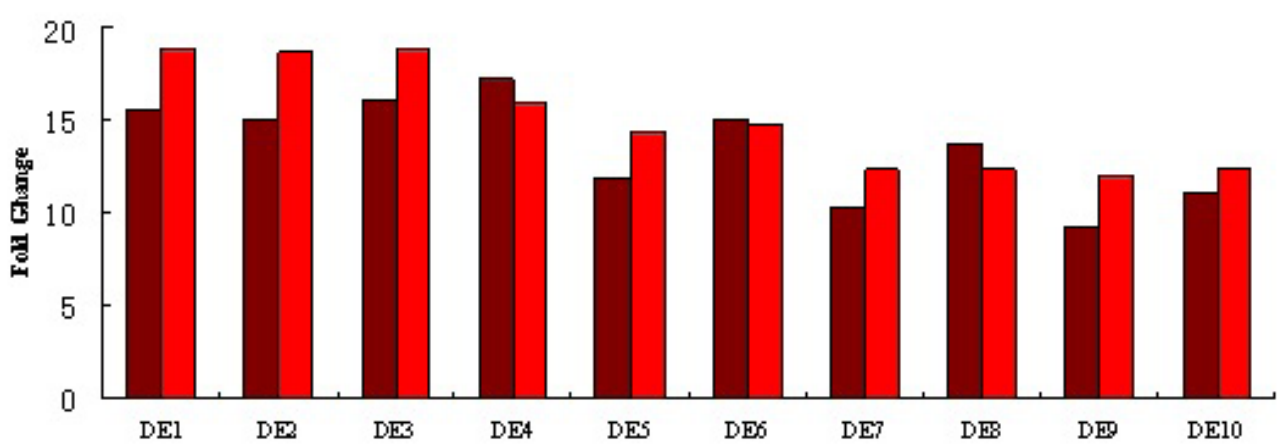

Figure 7. Relative expression levels obtained by real time PCR and transcriptome analyses of 10 candidate genes. Abscissa represents differentially expressed candidate genes; ordinates represent fold change ( $\log _{2}$ ratio).

Functional annotations were assigned to 4010 of the DEGs (95.86\%). DEGs between the mutant and the control were involved in the processing of genetic information, metabolism, transcription, translation, and signal transduction related to major substances such as sugars, fats, amino acids, and plant hormones. The results indicated that gene expression was affected by many factors, and that the transfer of genetic information at the different growth stages of soybean is a very complicated process. In addition, we found there was about a $40 \%$ overlap in the expressed DEGs associated with peptidase activity and metal ion binding. In this study, the smallest number of DEGs was found between the young pod mutant and the young pod control. The expression level of most genes was relatively low in the mutant at the young pod growth stage, and the foldchanges in expression were lower than those detected at other growth stages. Further research should be conducted to explore the reasons for the low levels of gene expression observed in the young pod of the mutant.

The main objective of plant transcriptome sequencing is to evaluate variations in gene expression under different conditions or among different growth stages, and to identify new genes or those specifically expressed during a particular period via comparisons of sequences with those in databases. For example, transcriptome sequencing of soybean leaves and roots identified many genes that were expressed under different salinity and drought stress conditions (Fan et al., 2013). Similarly, transcriptome sequencing analyses of cucumber female flowers and bisexual flowers identified genes related to sex differentiation of cucumber flowers (Guo et al., 2010). In this study, we compared DEGs between the soybean four-seed pod mutant and the control at three different developmental stages using transcriptome analysis. There were 206 up-regulated genes with $\log _{2}$ ratio value greater than 3 , including 103 genes with functional annotations. GO analysis showed that these up-regulated genes were involved in catalytic reactions, transcript level regulation, modulation of enzyme regulator activity, primary and secondary metabolism, tolerance responses, and other related biological metabolic pathways. Among these 103 genes, the most abundant were those encoding products related to protein binding/DNA binding/nucleotide binding $(36 \%)$, followed by those involved in metal ion binding (15\%). A previous study showed that the leaf photosynthetic rate and the accumulation of photosynthetic products increased in transgenic tobacco plants expressing the soybean ferritin gene (Wang, 2010). That study also showed that 
the lack of ferritin genes inhibits vegetative growth and the reproductive ability of the plant. Based on the results of the present study, we tentatively suggest that the up-regulated DEGs related to protein binding, DNA binding, nucleotide binding and metal ion binding may be related to the formation of four-seed pods or yield enhancement in soybean.

In this study, eight significantly enriched metabolic pathways (Q_value $\leq 0.05$ ) in three different growth periods were identified. These pathways included those related to amino acid synthesis and metabolism, carbon metabolism, plant hormone signal transduction, and protein processing in the endoplasmic reticulum. We conclude that these pathways may be related to the formation of four- or multiple-seed pods. These results lay the foundation for further research on the molecular mechanism of the formation of four- or multiple-seed pods at the transcriptome level.

\section{Conflicts of interest}

The authors declare no conflict of interest.

\section{ACKNOWLEDGMENTS}

The authors wish to thank Professor Zenglu Li (Center for Applied Genetic Technologies, University of Georgia, GA, USA) for his repair and advice. Research supported by the Education Department of Jilin province (\#2015-191), the Agency of Science and Technology of Jilin Provincial Science \& Technology Department (\#20140204021NY and \#20140101015JC), and the Campus Startup Funds of Jilin Agricultural University (\#201242).

\section{REFERENCES}

Cannon SB, May GD and Jackson SA (2009). Three sequenced legume genomes and many crop species: rich opportunities for translational genomics. Plant Physiol. 151: 970-977.

Chan C, Qi XP, Li MW, Wong FL, et al. (2012). Recent developments of genomic research in soybean. J. Genet. Genomics 39: 317-324.

Cheng LB, Li SY and He GY (2009). Isolation and expression profile analysis of genes relevant to chilling stress during seed imbibition in soybean [Glycine max (L.) Meer.]. Agric. Sci. China 8: 521-528.

Fan XD, Wang JQ, Yang N, Dong YY, et al. (2013). Gene expression profiling of soybean leaves and roots under salt, salinealkali and drought stress by high-throughput Illumina sequencing. Gene 512: 392-402.

Graham PH and Vance CP (2003). Legumes: importance and constraints to greater use. Plant Physiol. 131: 872-877.

Guo SG, Zheng Y, Joung JG, Liu SQ, et al. (2010). Transcriptome sequencing and comparative analysis of cucumber flowers with different sex types. BMC Genomics 11: 384.

Jain M (2012). Next-generation sequencing technologies for gene expression profiling in plants. Brief. Funct. Genomics 11: 63-70.

Jain M, Misra G, Patel RK, Priya P, et al. (2013). A draft genome sequence of the pulse crop chickpea (Cicer arietinum L.). Plant J. 74: 715-729.

Jung $\mathrm{CH}$, Wong CE, Singh MB and Bhalla PL (2012). Comparative genomic analysis of soybean flowering genes. PLoS One 7: e38250.

Kenneth JL and Thomas DS (2001). Analysis of relative gene expression data using real-time quantitative PCR and the $2^{-\Delta \Delta C t}$ method. Methods 25: 402-408.

Lulin H, Xiao Y, Pei S, Wen T, et al. (2012). The first Illumina-based de novo transcriptome sequencing and analysis of safflower flowers. PLoS One 7: e38653.

Margulies M, Egholm M, Altman WE, Attiya S, et al. (2005). Genome sequencing in microfabricated high-density picolitre reactors. Nature 437: 376-380.

Mortazavi A, Williams BA, McCue K, Schaeffer L, et al. (2008). Mapping and quantifying mammalian transcriptomes by RNASeq. Nat. Methods 5: 621-628. 
Ozsolak F and Milos PM (2011). RNA sequencing: advances, challenges and opportunities. Nat. Rev. Genet. $12: 87-98$.

Peng YH, Zhu JC, Yang GB and Yuan JZ (1994). Relation of soybean leaf shape distribution to 4-seeded pods. Acta Agron. Sin. 20: 501-503.

Sato S, Nakamura Y, Kaneko T, Asamizu E, et al. (2008). Genome structure of the legume, Lotus japonicus. DNA Res. 15: 227-239.

Schmutz J, Cannon SB, Schlueter J, Ma JX, et al. (2010). Genome sequence of the palaeopolyploid soybean. Nature 463: 178-183.

Severin AJ, Woody JL, Bolon YT, Joseph B, et al. (2010). RNA-Seq Atlas of Glycine max: A guide to the soybean transcriptome. BMC Plant Biol. 10: 160.

Soybean Growth and Development [http://www.soybeanmanagement.info].

Tatusov RL, Fedorova ND, Jackson JD, Jacobs AR, et al. (2003). The COG database: an updated version includes eukaryotes. BMC Bioinformatics 4: 41.

Young ND, Debellé F, Oldroyd GE, Geurts R, et al. (2011). The Medicago genome provides insight into the evolution of rhizobial symbioses. Nature 480: 520-524.

Varshney RK, Chen W, Li Y, Bharti AK, et al. (2011). Draft genome sequence of pigeonpea (Cajanus cajan), an orphan legume crop of resource-poor farmers. Nat. Biotechnol. 30: 83-89.

Varshney RK, Song C, Saxena RK, Azam S, et al. (2013). Draft genome sequence of chickpea (Cicer arietinum) provides a resource for trait improvement. Nat. Biotechnol. 31: 240-246.

Wang XZ, Zhang XJ, Zhou R, Sha AH, et al. (2007). QTL analysis of seed and pod traits in soybean RIL population. Acta Agron. Sin. 33: 441-448.

Wang Z, Gerstein M and Snyder M (2009). RNA-Seq: a revolutionary tool for transcriptomics. Nat. Rev. Genet. 10 : 57-63.

Wang SL (2010). Cloning of soybean Fettitin gene, construction of its expression vector and transformation with Arabidopsis. $\mathrm{PhD}$ thesis, Henan Agricultural University, Biotechnology Department.

Wong CE, Singh MB and Bhalla PL (2009). Molecular processes underlying the floral transition in the soybean shoot apical meristem. Plant J. 57: 832-845.

Wong CE, Singh MB and Bhalla PL (2013). The dynamics of soybean leaf and shoot apical meristem transcriptome undergoing floral initiation process. PLoS One 8: e65319.

Zhai FL (1988). Crop Quality Breeding. China Agricultural Press, Beijing.

Zhou XA, Wang XZ, Wu XJ, Cai SP, et al. (2005). Relation of three-seed and four-seed pods with yield of RIL in soybeans. Chin. J. Oil Crop Sci. 27: 22-25 